\title{
On the Maximum Achievable Rates in Wireless Multicast Networks
}

\author{
Seyed Reza Mirghaderi, Alireza Bayesteh and Amir K. Khandani \\ Coding and Signal Transmission Group \\ Electrical \& Computer Engineering Department \\ University of Waterloo, Ontario \\ Canada, ON N2L 3G1 \\ \{smirghad, alireza, khandani\}@cst.uwaterloo.ca
}

\begin{abstract}
A wireless multicast network with a stringent decoding delay constraint and a minimum required multicast data rate is characterized. Assuming the channel state information is available only at the receiver sides, and a single antenna system, the optimal expected rate achievable by a random user in the network is derived in terms of the minimum multicast requirement in two scenarios: hard coverage constraint and soft coverage constraint. In the first case, the minimum multicast requirement is expressed by multicast outage capacity while in the second case, the expected multicast rate should satisfy the minimum requirements. Also, the optimum power allocation in an infinite layer superposition code, achieving the highest expected typical rate, is derived subject to the coverage constraints. For the MISO case, a suboptimal coding scheme is proposed, which is shown to be asymptotically optimal, when the number of transmit antennas grows at least logarithmically with the number of users in the network ${ }^{1}$.
\end{abstract}

\section{INTRODUCTION}

In a wireless multicast system, a common source is transmitted to $N$ users, through a fading channel. In such networks, two issues can be studied as a measure of performance: network coverage and quality of service. In the first case, the objective is to cover all the nodes in the network at least with a basic service, regardless of their channel quality. From this point of view, all the users have basically the same opportunity to receive data. However, in the second case, the average quality of service is the main objective. Therefore, users with better channel status should receive higher data rates and consequently, better quality of service. The coverage issue in such systems is generally addressed as multicast minimum requirement.

Multicasting has been recently studied as a special scenario in broadcasting, where all the users are listening to a common source. In [2], the system challenges in lossy broadcasting of a common source are studied from information theoretical point of view. For an analog Gaussian source with a bandwidth equal to the channel bandwidth, analog transmission achieves the minimum average end-to-end distortion. The scenario in which the source has a larger bandwidth is studied in [3], where different methods of digital transmission are investigated.

\footnotetext{
${ }^{1}$ This work is financially supported by Nortel and by matching funds from the federal government of Canada (NSERC) and province of Ontario (OCE).
}

Since the performance of a multicast network is strongly affected by the user with the worst channel condition, we are motivated to define a more fair approach. We consider a wireless multicast network in a slowly fading Gaussian environment. The objective is to maximize the average performance while a multicast constraint is satisfied. Average performance is defined as the service received by a randomly chosen user (typical user) in the network, while the multicast requirement is the service received by all the users. These two requirements in a multicast network define a tradeoff, since the first one deals with a typical user of the network while the second depends on the worst channel state in the system. We assume the transmission block is large enough to yield a reliable communication. However, averaging over time is not possible because of the delay constraint. In other words, all the symbols within a transmission block experience the same channel gain. The channel state information (CSI) of each user is assumed to be known only at the receiver end. In this case, the ergodic capacity is not defined since the channel dose not have an ergodic behavior. The outage capacity [9] is defined for such channels as the maximum rate of single layered data, decodable with a high probability. In [6], a broadcast approach for a single user channel with these assumptions is proposed which optimizes the expected decodable rate. We will apply both "outage capacity" and "expected rate" definitions to characterize our network. Outage capacity is exploited when we have a hard coverage constraint on multicast data. In this case, we want to assure that a specific amount of data is conveyed within one transmission block to all the users, with a high probability. We relax the coverage constraint by stating it in terms of the expected delivered rate to all the users within one block. In both cases we maximize the expected typical rate.

This minimum-service based approach has been studied in [4] for a single user fading channel, assuming CSI is known at the transmitter. In that work, given a service outage constraint for a real time application, the average rate is maximized for a non real time application sent on top of it.

We will investigate the proposed multicast system in both SISO and MISO cases. The MISO multicast asymptotical capacity limits are examined in [5], when the CSI is available at the transmitter. It is shown that the adverse impact of the 
large number of users on the multicast capacity could be compensated by increasing the number of transmit antennas. We will study a similar scenario in our network and explore the effect of using multiple antennas.

The rest of this paper is organized as follows: in section II, the system model is elaborated. Section III and IV are specified to characterization of multicast network when we have a single antenna at the transmitter and at each receiver. In section III, we evaluate the optimal performance of the network in terms of the achievable expected typical rate and the multicast outage capacity. In other words, this section describes the hard multicast coverage constraint scenario. Section IV corresponds to a soft multicast coverage constraint, where expected multicast rate decoded in a block should satisfy the minimum requirement. In this scenario, we will explore the achievable expected typical rate. Section $\mathrm{V}$ investigates the MISO case, where we derive the asymptotical capacity limits for the multicast network.

\section{SySTEM MODEL}

When a single-antenna transmitter sends a common data to $N$ single-antenna receivers, the received signal at the $j$ th receiver, denoted by $y_{j}$ can be written as

$$
y_{j}=s_{j} x+n_{j} .
$$

In the above equation, $\{x\}$ is the transmitted signal with the total average power constraint $E\left[x^{2}\right] \leq \mathscr{P},\left\{n_{j}\right\} \sim \mathcal{C N}(0,1)$ is the Additive White Gaussian Noise (AWGN) at this receiver, and $s_{j} \sim \mathcal{C N}(0,1)$ is the channel coefficient from the transmitter to the $j$ th receiver. Therefore, the channel gain $h_{j}=\left|s_{j}\right|^{2}$ has the following CDF:

$$
F_{j}(h)=1-e^{-h},
$$

and is assumed to be constant during the transmission block. The typical (average) channel of the multicast network is defined as the channel of a randomly selected user. Since all the channels are i.i.d., the typical channel gain distribution is identical to that of each channel, i.e.,

$$
F_{\text {typ }}(h)=F_{j}(h)=F(h) .
$$

Since all the $N$ channels are Gaussian and they receive a common signal, the multicast channel is equivalent to the worst channel in the network. Due to statistical independence of the channels, the gain of that user has the following distribution:

$$
\operatorname{Pr}\left\{\min _{i}\left(h_{i}\right)>h\right\}=\left(\operatorname{Pr}\left\{h_{i}>h\right\}\right)^{N}=e^{-N h} .
$$

As a result, we have:

$$
F_{\text {mul }}(h)=F_{\min _{i}\left(h_{i}\right)}(h)=1-e^{-N h} .
$$

In this paper, we are dealing with three measures defined in our network, as follows:

- the multicast outage rate, $R_{\epsilon}$, the rate decodable at the multicast channel with probability $(1-\epsilon)$,
- the expected multicast rate, $R_{m u l}=E_{h_{m u l}}[R(h)]$, where $h_{m u l}=\min _{i}\left(h_{i}\right)$, and $R(h)$ is the decodable data rate for the channel state $h$,

- the expected typical rate, $R_{\text {ave }}=E_{h_{t y p}}[R(h)]$.

\section{BROADCAST MODEL FOR AN UNKNOWN FADING CHANNEL}

In [6], a virtual broadcast model is proposed to optimize the expected rate of a SISO channel with a stringent decoding delay constraint. In this paper, we exploit the same model for a multicast channel in a more general fashion. In the following, we will study this model in detail.

Because of a decoding delay constraint in our network, each receiver is experiencing a single fading level during the whole period of transmission. Hence, for any coding scheme, we have a function $R(h)$ which determines the data rate decoded in channel state $h$. Regarding the degraded nature of the Gaussian channels, this function is increasing. Therefore

$$
R(h)-R(h-\mathrm{d} h)=\mathrm{d} R_{h} \geq 0
$$

Consider an infinite number of differently indexed virtual receivers, such that receiver $r_{h}$ is experiencing a fading level between $h$ and $h+\mathrm{d} h$. With this assumption, $r_{h}$ is receiving all the data received by $r_{h-\mathrm{d} h}$ in addition to $\mathrm{d} R_{h}$. The virtual receivers introduce a degraded broadcast network in which the rate associated to the virtual user $r_{h}$ is $\mathrm{d} R_{h}$. The actual user selects receiver $r_{h}$ with probability $f(h) \mathrm{d} h$, where $f(h)$ is the channel gain distribution function.

With this interpretation, for a given coding scheme, the distinction between different channels is their different probability distribution of virtual antenna selection. Hence, provided that both multicast and typical channel deal with the same signalling, all the measures defined in the previous section could be written in this setting:

$$
\begin{gathered}
R_{\text {ave }}=\int_{0}^{\infty} R(h) f(h) \mathrm{d} h=\int_{0}^{\infty}(1-F(h)) \mathrm{d} R_{h}, \\
R_{m u l}=\int_{0}^{\infty}\left(1-F_{m u l}(h)\right) \mathrm{d} R_{h}, \\
R_{\epsilon}=R\left(h_{\epsilon}\right)=\int_{0}^{h_{\epsilon}} \mathrm{d} R_{h},
\end{gathered}
$$

where $h_{\epsilon}=F_{m u l}^{-1}(\epsilon)$. The first two derivations are statistical averaging of decoded rate over different selected antennas. In the case of multicast channel, the selected antenna has a channel level lower than $h_{\epsilon}$ with probability $\epsilon$, and hence, the highest decodable rate with probability $1-\epsilon$ is $R\left(h_{\epsilon}\right)$. As seen above, the performance measures in our network are three different positive weighted sum-rates of the virtual broadcast network which forms a performance vector. We are always interested to achieve the optimal performance vector. In the following, we will propose a search space for the virtual broadcast rate vector which results in the optimal performance. Before that, we should give a definition for the optimality of a performance vector.

Deffinition 1 The boundary set $B_{1}$ of a closed convex region $R_{1} \subset R^{+^{n}}$, is defined as

$$
B_{1}=\left\{x \in R_{1} \mid \nexists x^{\prime} \in R^{++n}, x^{\prime} \neq \mathbf{0}, x+x^{\prime} \in R_{1}\right\}
$$


where $R^{+}$and $R^{++}$are the set of nonnegative and strictly positive real numbers, respectively.

With the above definition, a performance vector is optimal if it is in the boundary set of all possible performance vectors.

Lemma 1 Consider a mapping function $g($.$) from a closed$ region $R_{1} \subset R^{+^{n}}$ to $R_{2} \subset R^{+^{k}}$, such that $g(x)=M x$, where $M \in R^{+^{k}} \times R^{+^{n}}$. Denote $B_{1}$ and $B_{2}$ the boundary sets of regions $R_{1}$ and $R_{2}$, respectively. We have

$$
B_{2} \subset g\left(B_{1}\right)
$$

The proof is given in [10].

Corollary 1 Given two positive weighted sum rate of a virtual broadcast channel, $R_{w_{1}}=\int w_{1}(h) \mathrm{d} R_{h}$ and $R_{w_{2}}=$ $\int w_{2}(h) \mathrm{d} R_{h}$, for any vector $\mathbf{v}$ in the boundary set of $\left(R_{w_{1}}, R_{w_{2}}\right)$, there exists a scalar positive function $\rho^{\mathbf{v}}(h)$ such that

$$
\mathrm{d} R_{h}^{\mathbf{v}}=\log \left(1+\frac{h \rho^{\mathbf{v}}(h) \mathrm{d} h}{1+h \int_{h}^{\infty} \rho^{\mathbf{v}}(u) \mathrm{d} u}\right)
$$

and $\mathbf{v}=\left[\int w_{1}(h) \mathrm{d} R_{h}^{\mathbf{v}}, \int w_{2}(h) \mathrm{d} R_{h}^{\mathbf{v}}\right]$, where $\int_{0}^{\infty} \rho^{\mathbf{v}}(u) \mathrm{d} u=$ $\mathscr{P}$.

The proof which is given in [10] is concluded from the above lemma and the fact that superposition coding achieves the capacity region of a degraded broadcast channel. We will use the above lemma in this paper to deduce that superposition coding achieves the optimal performance vector.

\section{Hard Coverage Constraint}

In this section, we consider a scenario where the multicast data has a high priority. Hence, it should be delivered to all the users in the network with a high probability $(1-\epsilon)$, where $\epsilon$ is the outage probability of the system. In this case, any loss of the multicast data by any user is defined as a coverage outage. Given this constraint, we want to maximize the average rate received by a randomly chosen user in the network. This average rate includes the expected rate of all data received by a typical user, even if the user is in outage. However, we will show that for a small enough outage probability, the users in the outage do not contribute to the expected average rate (it is optimum not to allocate them any power). In this scenario, we deal with two channels: (i) a multicast channel for which we want to guarantee an outage rate $R_{\epsilon}$, and (ii) an average channel for which the highest expected rate $R_{\text {ave }}$ is desired.

Setting $w_{1}(h)=1_{\left\{h \leq h_{\epsilon}\right\}}$ and $w_{2}(h)=1-F(h)$, Corollary 1 states that the boundary set of $\left(R_{\epsilon}, R_{\text {ave }}\right)$ is achieved by superposition coding:

$$
\mathrm{d} R_{h}=\log \left(1+\frac{h \rho(h) \mathrm{d} h}{1+h I(h)}\right)=\int_{I(h)}^{I(h)+\rho(h) \mathrm{d} h} \frac{h \mathrm{~d} p}{1+h p},
$$

where $I(h)=\int_{h}^{\infty} \rho(u) \mathrm{d} u$. Note that, $\mathrm{d} R_{h}$ is not necessarily very small since our power allocation function might have some impulses in the general case. As stated before, we want to jointly optimize the weighted sum of these rates according to the weighting functions $w_{1}(h)$ and $w_{2}(h)$. The optimization is on the function $I(h)$ and $\rho(h)$. However, we can simplify our optimization problem to a point optimization. Let us define $s(p)$ as

$$
s(p)=\max \{h \mid I(h) \geq p\} .
$$

It is evident that this function is a decreasing function of $p$. According to (4), we can write the expected rate as

$$
R_{\text {ave }}=\int_{0}^{\infty}(1-F(h)) \mathrm{d} R_{h}=\int_{0}^{\mathscr{P}} g(p, s(p)) \mathrm{d} p,
$$

where $g(x, y)=(1-F(y)) \frac{y}{1+x y}$. It is proved in [10] that

$$
\arg \max \left(\left.g(x, y)\right|_{x=p}\right)=I_{0}^{-1}(p),
$$

where $I_{0}(h)=\frac{(1-F(h))-h f(h)}{h^{2} f(h)}$. Moreover, $\left.g(x, y)\right|_{x=p}$ is increasing for $y<I_{0}^{-1}(p)$, and decreasing elsewhere.

Let us define $P_{\epsilon}^{s(.)}$ for the function $s($.$) as$

$$
P_{\epsilon}^{s(.)}=\min \left\{p \mid s(p) \leq h_{\epsilon}\right\},
$$

where $h_{\epsilon}=F_{m u l}^{-1}(\epsilon)$. For simplicity, we assume $h_{\epsilon} \leq 1$ (this assumption is justified in [10]). With the above definitions, our problem is translated to find

$$
\max _{s(.)} R_{\text {ave }}=\max _{s(.)} \int_{0}^{\mathscr{P}} g(p, s(p)) \mathrm{d} p,
$$

subject to $\int_{P_{\epsilon}^{s(.)}}^{\mathscr{P}} m(p, s(p)) \mathrm{d} p \geq R_{\epsilon}$, where $m(x, y)=\frac{y}{1+x y}$ and $s($.$) is constrained to be a decreasing positive function. For$ any chosen $x, m(x, y)$ is an increasing function of $y$. Hence, we can write

$$
R_{\epsilon} \leq \int_{P_{\epsilon}^{s(.)}}^{\mathscr{P}} m\left(p, h_{\epsilon}\right) \mathrm{d} p=\log \left(\frac{1+h_{\epsilon} \mathscr{P}}{1+h_{\epsilon} P_{\epsilon}^{s(.)}}\right)=C\left(P_{\epsilon}^{s(.)}\right) .
$$

Since $C(p)$ is a decreasing function of $p$,

$$
P_{\epsilon}^{s(.)} \leq C^{-1}\left(R_{\epsilon}\right) \text {. }
$$

Lemma 2 Denoting the optimizer of the problem (7) as $s^{*}($.$) ,$ we have $P_{\epsilon}^{s^{*}(.)} \leq I_{0}\left(h_{\epsilon}\right)$.

The proof is given in [10], considering the behavior of the function $g(x, y)$. The above lemma states the fact that, applying the multicast outage constraint, more power will be allocated to the channel gains lower than the outage threshold, compared to the unconstrained scenario [6], where $I_{0}($.$) is the$ interference term which leads to the optimal expected rate.

Lemma 3 Given $P_{\epsilon}^{s(.)}=\alpha$, the optimizer of (7) is given by

$$
s_{\alpha}^{*}(p)=\eta(\lambda, p)= \begin{cases}I_{0}^{-1}(p) & p<\alpha \\ h_{\epsilon} & \alpha \leq p \leq I_{\lambda}\left(h_{\epsilon}\right) \\ I_{\lambda}^{-1}(p) & p>I_{\lambda}\left(h_{\epsilon}\right)\end{cases}
$$

where $I_{\lambda}(h)=\frac{(\lambda+1-F(h))-h f(h)}{h^{2} f(h)}$, and

$$
\lambda=\left\{\begin{array}{l}
0, \int_{\alpha}^{\mathscr{P}} m(p, \eta(0, p)) \mathrm{d} p>R_{\epsilon} \\
\arg \left(\int_{\alpha}^{\mathscr{P}} m(p, \eta(\lambda, p)) \mathrm{d} p=R_{\epsilon}\right), \text { otherwise }
\end{array} .\right.
$$

The proof is given in [10]. 
Theorem 1 The solution to the optimization problem (7) can be written as

$$
\max R_{\text {ave }}=\max _{0 \leq \alpha \leq \min \left(C^{-1}\left(R_{\epsilon}\right), I_{0}\left(h_{\epsilon}\right)\right)} \int_{0}^{\mathscr{P}} g\left(p, s_{\alpha}^{*}(p)\right) \mathrm{d} p .
$$

Proof: The proof is directly concluded from Lemma 2, Lemma 3 , and inequality (8).

Corollary 2 The boundary set of $\left(R_{\epsilon}, R_{\text {ave }}\right)$, is given by $\left(C_{\epsilon}, C_{\text {ave }}\right)$, such that

$$
C_{\epsilon}=\log \left(1+\frac{h_{\epsilon} \beta \mathscr{P}}{1+h_{\epsilon}(1-\beta) \mathscr{P}}\right),
$$

where $\beta$ changes from 0 to 1 and

$$
C_{\text {ave }}=2\left(E_{i}(\theta(\beta))-E_{i}(1)\right)-\left(e^{-\theta(\beta)}-e^{-1}\right)+e^{-h_{\epsilon}} C_{\epsilon},
$$

where $\theta(\beta)=\frac{2}{1+\sqrt{1+4(1-\beta) \mathscr{P}}}$, and $E_{i}(x)=\int_{x}^{\infty} \frac{e^{-t}}{t} \mathrm{~d} t$, for any $\epsilon>0$ such that $h_{\epsilon} \leq I_{0}^{-1}(\mathscr{P})$.

The proof is given in [10].

An interesting conclusion of Corollary 2 is that, the expected typical rate is maximized when the multicast rate is provided in a single layer code. In the case we have no multicast constraint, it is shown in [6] that a multilevel coding with a small rate in each level is optimal in terms of maximizing the expected rate. However, when we are constrained to distribute a fraction of power to a set of low channel gains $\left[0, h_{\epsilon}\right]$ (coverage constraint), it is optimum to allocate all the power to the highest gain $\left(h_{\epsilon}\right)$.

Note that the assumption $h_{\epsilon} \leq I_{0}^{-1}(\mathscr{P})$ is not hard to satisfy, since the outage probability $\epsilon$ is usually small. Moreover, the value of $h_{\epsilon}$ decreases significantly with the number of users, such that it could be approximated by $\frac{\epsilon}{N}$ [10]. For example, for $N=5$ and $\mathscr{P}=100$, the outage probability $\epsilon$ could be as high as 0.38 in order to have $h_{\epsilon} \leq I_{0}^{-1}(\mathscr{P})$. In figure 1 we can see the capacity region of this network when $\epsilon=0.01$. It is evident that due to hard coverage constraint for all the users, the achievable outage rates are very small in comparison with the expected rate values.

\section{Soft Coverage Constraint}

In the previous section, we observed that a strict coverage constraint for multicasting results in very small values of multicast rate. We can relax the coverage requirement by considering the average service received by all the users in one channel block. In fact, we can replace the outage requirement by the expected multicast rate. In this case, all the users should receive a minimum rate in average and given that, we want a typical user to receive the highest expected rate. Therefore, the measures we are dealing with in this section are $R_{m u l}$ and $R_{\text {ave. }}$

Theorem 2 The boundary set of $\left(R_{m u l}, R_{a v e}\right)$, is given by $\left(C_{m u l}, C_{\text {ave }}\right)$, such that:

$$
\begin{aligned}
C_{\text {ave }} & =\int_{0}^{\infty} e^{-u \frac{u \rho_{\gamma}(u) \mathrm{d} u}{1+u I_{\gamma}(u)},} \\
C_{m u l} & =\int_{0}^{\infty} e^{-N u} \frac{u \rho_{\gamma}(u) \mathrm{d} u}{1+u I_{\gamma}(u)},
\end{aligned}
$$

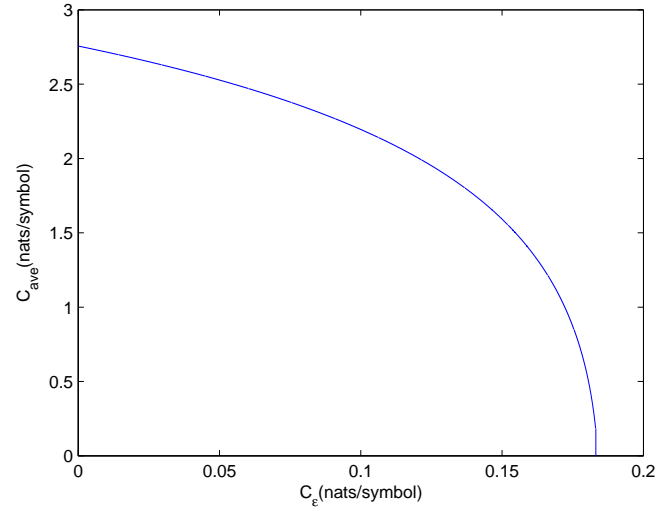

Fig. 1. Hard coverage constraint: multicast outage capacity vs. expected typical rate for $\mathscr{P}=100$ and $N=5$

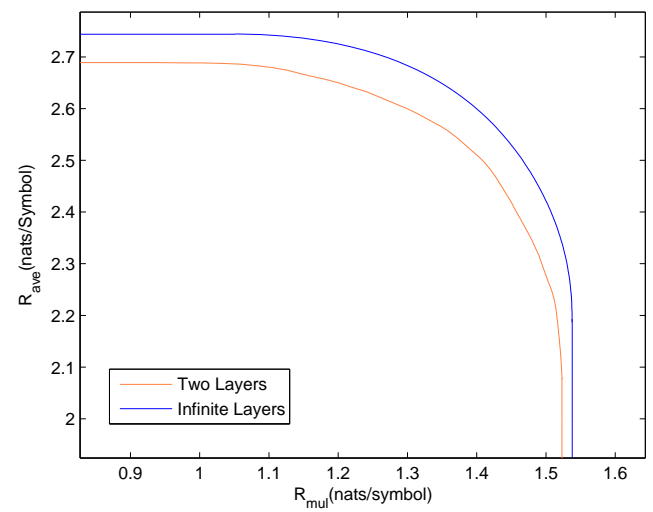

Fig. 2. Soft coverage constraint: expected multicast rate vs. expected typical rate for $\mathscr{P}=100$ and $N=5$

where

$$
I_{\gamma}(h)=\left\{\begin{array}{ll}
\mathscr{P} & \text { if } h<h_{0} \\
\frac{e^{-h}(1-h)+\gamma e^{-N h}(1-N h)}{h^{2}\left(e^{-h}+\gamma N e^{-N h}\right)} & h_{0}<h<h_{1} \\
0 & h>h_{1}
\end{array},\right.
$$

$\rho_{\gamma}(h)=-\frac{\partial I_{\gamma}(h)}{\partial h}$, and $h_{0}$ and $h_{1}$ are real numbers, such that

$$
\begin{aligned}
& \frac{e^{-h_{0}}\left(1-h_{0}\right)+\gamma e^{-N h_{0}}\left(1-N h_{0}\right)}{h_{0}^{2}\left(e^{-h_{0}}+\gamma N e^{-N h_{0}}\right)}=\mathscr{P}, \\
& \frac{e^{-h_{1}}\left(1-h_{1}\right)+\gamma e^{-N h_{1}}\left(1-N h_{1}\right)}{h_{1}^{2}\left(e^{-h_{1}}+\gamma N e^{-N h_{1}}\right)}=0,
\end{aligned}
$$

for different positive values of $\gamma$.

The proof is given in [10], setting $w_{1}(h)=1-F_{m u l}(h)$ and $w_{2}(h)=1-F(h)$ and using Corollary 1 .

Figure 2 shows the achievable rate region for $N=5$ and $\mathscr{P}=100$. It is shown in [7], that a good fraction of the highest expected rate with infinite layers of code is achieved by two layers. Figure 2 shows that this is true for our multicast network as well.

In figure 3, the optimal power distribution function is depicted for the multicast requirement $R_{m u l} \geq 1.4$ (nats/symbol) and is compared to the case we have no multicast constraint. 


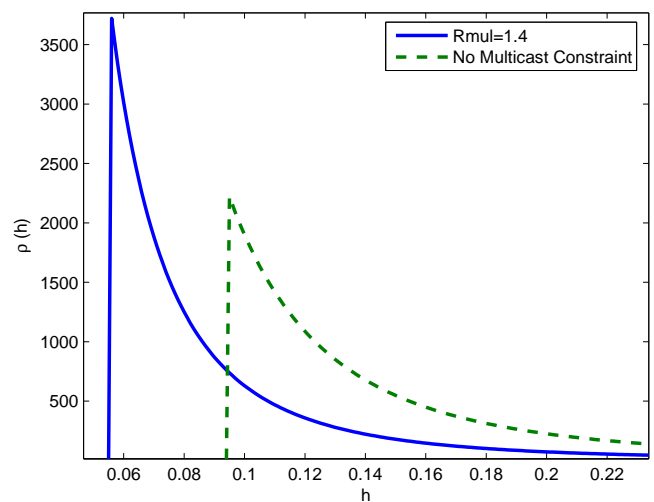

Fig. 3. Power distribution function $\rho(h)$ for no multicast requirement and for $R_{m u l}=1.4$ nats/symbol

As expected, the coverage constraint for all the users has shifted the power to lower channel gains, in order to provide service for the user with the worst channel quality in the network.

\section{EXTENSION TO MISO}

In the case we have multiple $(M)$ antennas at the transmitter, we can adopt the broadcast approach proposed in [6]. In this approach, the receiver with unknown quasi-static fading MISO channel is modeled as a continuum of receivers each associated with a channel realization. These receivers are ordered in a degraded fashion, based on their normalized channel norm, $\frac{\left\|H H^{\dagger}\right\|}{M}$. Using this model, we can convert the MISO case to the previously studied SISO network with the channel fading level $\frac{1}{M}\left\|H_{i} H_{i}^{\dagger}\right\|$, corresponding to the user $i$. Hence, all the results of the previous sections can be obtained, by using an equivalent SISO channel with distribution

$$
F_{\text {typ }}(h)=F_{\frac{1}{M}\left\|H_{i} H_{i}^{\dagger}\right\|}(h)=1-\frac{\Gamma(M, M h)}{\Gamma(M)},
$$

and multicast channel distribution

$$
F_{\text {mul }}(h)=\operatorname{Pr}\left\{\min _{i} \frac{1}{M}\left\|H_{i} H_{i}^{\dagger}\right\|>h\right\}=\left(\frac{\Gamma(M, M h)}{\Gamma(M)}\right)^{N} .
$$

where $\Gamma(\alpha)$ is a gamma function, and $\Gamma(\alpha, \beta)$ is an upper incomplete gamma function. Further analytical results are presented in [10].

Since MIMO-BC is inherently non-degraded, this approach dose not necessarily lead to the optimum performance. However, we will show that it is optimal when $M$ is large enough compared to number of users. Above that, we will prove that one can compensate the adverse effect of increasing $N$ on multicast rate (as observed in figure 4), by using more transmit antennas. This is sensible, since we are using multiple independent paths to convey the data, so the probability of having very low channel gains for all paths (which mainly affects the capacity of the multicast channel) significantly decreases. The above facts are true for both coverage constraint scenarios. However, due to space limitation, we only study the

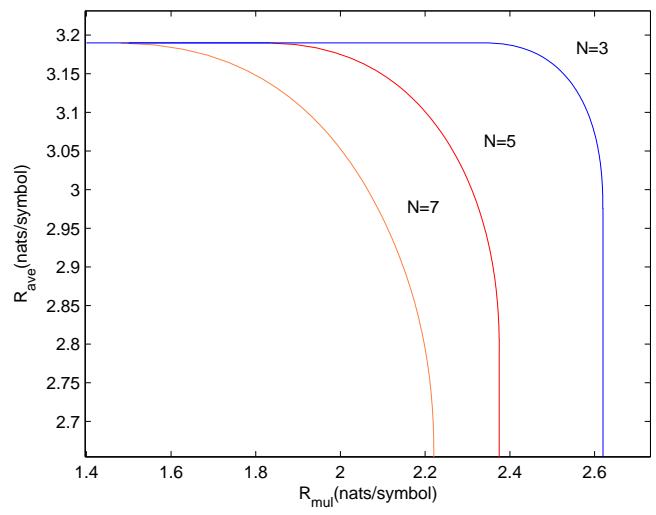

Fig. 4. Soft coverage constraint: MISO expected multicast rate vs. expected typical rate for different number of users, $M=2$ and $\mathscr{P}=100$

case with a soft coverage constraint in the following theorem:

Theorem 3 For large values of $M$ and $N$, the proposed infinite layer superposition coding will provide $R_{m u l}$, such that $R_{m u l} \geq R_{o p t}-\epsilon$, if

$$
M>\frac{\mathscr{P}^{2} \log (N)+\omega(1)}{(1+\mathscr{P})^{2} \epsilon^{2}},
$$

where $R_{o p t}$ is the highest achievable average rate for a randomly selected user in the network and $\epsilon$ is an arbitrarily small positive number.

The proof is given in [10].

\section{REFERENCES}

[1] T. Cover and J. Thomas, Elements of Information Theory. New York: Wiley, 1991

[2] S. Sesia, G. Caire, and G. Vivier, "Broadcasting a common source: Information thoeretic results and system challenges," in Proc. IEEE Int. Symp. Information Theory (ISIT 03), Monte Verita, Switzerland, Feb. 24-27, 2003

[3] S. Sesia, G. Caire, and G. Vivier, "Lossy Transmission over SlowFading AWGN Channels: a Comparison of Progressive, Superposition and Hybrid Approaches," in Proc. IEEE Int. Symp. Information Theory (ISIT 05), Adelaide, Australia, Sep. 4-9, 2005, p. 224-228

[4] Jianghong Luo, Roy Yates and Predrag Spasojevic, "Service Outage Based Power and Rate Allocation for Parallel Fading Channels," IEEE Trans. Inf. Theory, vol. 51, no. 7, pp. 2594-2611, Jul. 2005.

[5] N. Jindal and Z. Q. Luo, "Capacity Limits of Multiple Antenna Multicast, " in Proc. IEEE Int. Symp. Information Theory (ISIT 06), Seatle, USA, July 9-14 2006.

[6] S. Shamai (Shitz) and A. Steiner, "A broadcast approach for a singleuser slowly fading MIMO channel" IEEE Trans. Inf. Theory, vol. 49, no. 10, pp. 2617-2635, Oct. 2003.

[7] Y. Liu, K. N. Lau, C. Y. Takeshita, and M. P. Fitz, "Optimal rate allocation for superposition coding in quasistatic fading channels," in Proc. IEEE Int. Symp. Information Theory (ISIT 02), Lausanne, Switzerland, June 30-July 5 2002, p. 111.

[8] P. Bergmans and T. Cover, "Cooperative Broadcasting," IEEE Trans. Inf. Theory, Volume 20, Issue 3, May 1974, pp. 317-324.

[9] M. Effros and A. Goldsmith, "Capacity of general channels with receiver side information," in Proc. 1998 IEEE Int. Symp. Information Theory (Cambridge, MA, Aug. 1721, 1998), p. 39.

[10] R. Mirghaderi,A. Bayesteh, and A. Khandani , "On the capacity of wireless multicast networks," UW Technical Report \#2006-25, Nov. 2006.(available at http://cst.uwaterloo.ca/ $\sim$ smirghad/multicast.pdf) 